\section{Molecular turnover and memory}

SIR - Francis Crick considers in News and Views under the title "Memory and molecular turnover" " the problem, often overlooked, that although memory operates over periods of years or decades, most macromolecules (with the exception of DNA) turn over with half lives of hours or weeks. Crick sees the dilemma since memory is prolonged and a consequence of inter-synaptic interaction which is dependent on fixed intrasynaptic macromolecules, such as membrane glycoproteins, either singly or more probably in larger aggregates of some form. To sustain memory two alternative strategies are proposed, either the memory macromolecule is immune from turnover (a less likely possibility for Crick) or the memory macromolecules in a synapse can be replaced one at a time without altering the overall state of the memory macromolecular complex.

My response to Crick's interesting challenge lies in the observation that perinuclear (often sided) membrane disposition is required before protein catabolism in normal cells takes place ${ }^{2}$ and some special association of proteins with cytoskeletal elements may precede routing to the cellular destructive machinery ${ }^{3,4}$.

Nerve cells are exquisitely polarized with the cell body (nucleus, polysomes and Golgi) quite spatially distinct from synapse forming processes such as axons or dendrites. Therefore, memory proteins dispatched from around the nucleus into processes by axoplasmic flow would need to retrace their steps for destruction as described in the protein turnover cycle ${ }^{s}$. It is not at all fanciful to suppose that the intricate arborizations of nerve cell processes in the temporal cortex (and everywhere else) have evolved, at least in part, in order to separate and 'immunize' informational macromolecules spatially from the apparatus of molecular turnover. Simply detaching such macromolecules from the neuroskeletal system would suffice (compare refs 2-4), thereby preventing (or slowing) the return of the macromolecules to the perinuclear destructive machinery. Alternatively, selective reversible detachment-attachment to the neuroskeleton would identify populations of proteins (or individual proteins) which are to be routed for destruction and replacement.

Both of the above alternatives could be mediated by protein modifications such as those described by Crick. Likewise both possibilities could operate together in a single neurone. Either way, neuronal evolution could have capitalized on the spatial separation of proteins (in membranes) in the synapses of cellular processes away from the perinuclear destruction apparatus in cell bodies so that information storage is achieved by permanent or temporary macromolecular stabilization.

It is perhaps salutory in an ageing human population that facultative loss, as occurs in Alzheimer's disease, and motor disorder, as seen in Parkinson's disease, might be triggered by the disruption of the status of cell body-synaptic trafficking in cortical neurones and nigro-striated pathways respectively, leading to intraneuronal degradative (degenerative) processes.

\section{Department of Biochemistry,}

R.J. MAYER

The Medical School,

Queen's Medical Centre,

Clifton Boulevard,

Nottingham NG7 2UH, UK

1. Crick, F. Nature 312, 101 (1984).

2. Evans, P.J. \& Mayer, R.J. Biochem. J. 216, 151-161 (1983). 3. Rogers, S.W. \& Rechsteiner, M. in Intracellular Protein Catabolism (eds Bond, J.S., Bird, J.W.C. \& Khairallah, E.) (Liss, New York, in the press).

4. Doherty, F. \& Mayer, R.J. Biochem. J. (in the press).

5. Mayer, R.J., Evans, P., Russel, S. \& Amenta, J.S. Ciba Fdn Symp. 103, 202-219 (1984)

\section{Human B-cell cytotoxic lymphokine priority}

SIR - The recent article on the cloning and expression of human lymphotoxin by scientists from Genentech Inc. ${ }^{1}$ described work that first came to my attention when a report appeared in The Guardian of 6 June 1984 concerning an announcement by the company that they had developed a new cancer drug which did not have side effects. The initial scientific papers from Genentech $^{2}$ stated that the lymphotoxin in question had a relative molecular mass $\left(M_{r}\right)$ of approximately 20,000 . I therefore thought that it must be a new cytotoxic lymphokine. Some seven years earlier ${ }^{3,4}$, I had published a description of a humoral cytotoxic factor produced by a human lymphoblastoid cell line of B-cell lineage derived from a local patient with leukaemia.

Those papers of mine, which characterized the properties of the factor and how it was distinguished from other forms of cell killing, were probably the first well documented studies showing that some human B-lymphoblasts growing in vitro produced a cytotoxic lymphokine. In these early papers we also reported that the humoral factor preferentially kills malignant cells and that it had reduced by approximately 50 per cent the incidence of malignancy (fibrosarcoma) in mice ${ }^{3,4}$. Our studies also showed that the cytotoxic factor was a protein with an $M_{\mathrm{r}}$ of $65,000 \pm 1,000^{5}$.

In the recent paper in Nature by Genentech, the authors state that their initial published result (1984) on the relative molecular mass was incorrect and that the actual $M_{\mathrm{r}}$ of this human lymphoblastoid cell-derived lymphotoxin is $60,000-70,000$. This value is so closely similar to the 65,000 $M_{\mathrm{r}}$ we had previously reported in $1980^{5}$, that it now seems extremely likely that the human B-lymphoblast-produced lympho- toxin - cloned recently by Genentech is identical with the cytotoxic factor first described in $1977^{3,4}$. It will be for future studies to establish whether the cytotoxic factor described from Cambridge in 1977 and the lymphotoxin described in 1984 in the United States are in fact identical and to explore the efficacy in the treatment of malignant states.

A. KarPaS

Department of Haematological Medicine, University of Cambridge

Clinical School.

Hills Road,

Cambridge CB2 2QL, UK

1. Gray, P.W. et al. Nature 312, 721 (1984).

2. Aggarwal, B. B. et al. J. biol. Chem. 259, 686 (1984).

3. Karpas, A. Brit. J. Cancer 35, 152 (1977)

4. Karpas, A. Brit. J. Cancer 36, 437 (1977)

5. Neumann, H. \& Karpas, A. Bioch. J. 194, 847 (1981).

\section{HTLV-III, LAV, ARV are variants of same AIDS virus}

SIR - Retroviruses have been isolated reproducibly from patients with the acquired immunodeficiency syndrome (AIDS), and have been designated human T-cell lymphotropic virus (HTLV) type III $^{1}$, lymphadenopathy-associated virus $(\mathrm{LAV})^{2}$, or rather recently AIDS-related virus $(A R V)^{3}$ by different groups of investigators. Forty-eight independent HTLV-III isolates were originally reported from our laboratory ${ }^{1}$, several additional ones since ${ }^{4,5}$, and now we have obtained more than 100 independent isolates (S.Z. Sakhuddin et al., in preparation). The recent publications of the complete nucleotide sequence of two HTLV-III proviruses ${ }^{6}, \mathrm{LAV}^{7}$, and $\mathrm{ARV}^{8}$ allows a detailed comparison (see table). Sequences of HTLV-III clones $\mathrm{BH} 5$ and $\mathrm{BH} 8$ (representing the $5^{\prime}$ and $3^{\prime}$ portions of provirus(es), respectively), clone LAV1a, and ARV-2 are compared to HTLV-III clone BH10. LAV is closely related to HTLV-III clone $\mathrm{BH} 10$ and differs in $1.5 \%$ of nucleotides and $2.2 \%$ of amino acids, while ARV-2 differs in $6.3 \%$ of its base pairs and $9.2 \%$ of its amino acids from that of HTLV-III clone BH10. These data show that HTLV-III, LAV, and ARV are variants of the same virus. The greater sequence divergence of ARV from HTLV-III is not likely to be a result of errors in sequence determination. First, sequences obtained independently in different laboratories for the same HTLV-III clones were in agreement ${ }^{6}$. Second, multiple clones of ARV isolated from the same cell line infected with a virus isolate from a single individual differ in sequence from one another by only 2 or 3 base pairs $(b p)^{8}$. Third, we have sequenced another proviral clone of HTLV-III derived from another one (RF) of the original 48 isolates reported $^{1}$, which differs from $\mathrm{BH} 10$ to a similar degree as does ARV (our unpublished observations with B. Starcich, B. Hahn 